\title{
Association Between High-Flow Nasal Cannula and End-Expiratory Esophageal Pressures in Premature Infants
}

\author{
Narayan P Iyer MD and Maroun J Mhanna MD MPH
}

\begin{abstract}
BACKGROUND: High-flow nasal cannula (HFNC) is commonly being used to provide noninvasive respiratory support in newborn infants. Continuous distending pressure generated by these devices in preterm infants has not been elucidated. The aim of this work was to determine the association between $\mathrm{HFNC}$ flow in $\mathrm{L} / \mathrm{min}$ and end-expiratory esophageal pressure in $\mathrm{cm}_{2} \mathrm{O}$ in premature infants. METHODS: In a prospective observational study, end-expiratory esophageal pressure measurements were conducted in clinically stable premature infants who required HFNC. The end-expiratory esophageal pressure generated by the HFNC was measured by a regular feeding tube overlying the lower esophagus and connected to a pressure transducer. Each infant had different end-expiratory esophageal pressure measurements at different high flows ranging from 2 to $8 \mathrm{~L} / \mathrm{min}$. RESULTS: Nineteen premature infants were studied. Their birthweight (mean \pm SD) was $904 \pm 574 \mathrm{~g}$, and gestational age was $26.6 \pm 3.1$ weeks. Overall, 93 end-expiratory esophageal pressure measurements were conducted. At the time of the end-expiratory esophageal pressure measurements, infants were 1,458 $\pm 828 \mathrm{~g}$ and $7.2 \pm 4.8$ weeks old. At HFNC flows ranging from 2 to $8 \mathrm{~L} / \mathrm{min}$, the corresponding esophageal pressures ranged from 2 to $15 \mathrm{~cm} \mathrm{H}_{2} \mathrm{O}$. There was a statistically significant association between esophageal pressures and flows. In a linear regression model with the end-expiratory esophageal pressure as the dependent variable and HFNC flow as the independent variable, there was a significant correlation between the 2 variables: end-expiratory esophageal pressure $\left(\mathrm{cm} \mathrm{H}_{2} \mathrm{O}\right)=1.18 \times \mathrm{HFNC}(\mathrm{L} / \mathrm{min})\left(\mathrm{r}^{2}=0.95, P<.001\right)$. CONCLUSIONS: HFNC-associated end-expiratory esophageal pressure is measurable in premature infants. There is a significant association between flows and generated esophageal pressures. There is also variability in the amount of end-expiratory esophageal pressure generated. These observations should be kept in mind when using high HFNC flows in preterm infants. Key words: very low birthweight; prematurity; infants; high-flow nasal cannula; end-expiratory esophageal pressures. [Respir Care 2016;61(3):285-290. ㅇ 2016 Daedalus Enterprises]
\end{abstract}

\section{Introduction}

Noninvasive respiratory support has gained ground in recent years as an important tool to reduce long-term respiratory morbidity in preterm infants. Noninvasive respi-

\footnotetext{
The authors are affiliated with the Department of Pediatrics, MetroHealth Medical Center, Case Western Reserve University, Cleveland, Ohio.

The authors have disclosed no conflicts of interest.
}

Correspondence: Maroun J Mhanna MD MPH, Department of Pediatrics, MetroHealth Medical Center, 2500 MetroHealth Drive, Cleveland, OH 44109. E-mail: mmhanna@metrohealth.org.

DOI: $10.4187 /$ respcare. 04317 ratory support is usually provided by devices that provide pressure (ie, nasal CPAP) or high flow $(>2 \mathrm{~L} / \mathrm{min}) .{ }^{1} \mathrm{De}-$ vices using high-flow nasal cannula (HFNC) improve efficiency of breathing by injecting fresh heated humidified gas, thereby reducing the nasopharyngeal dead space. ${ }^{2}$ HFNC also provides a continuous distending pressure, and it has been shown to be comparable with distending pressure produced by nasal CPAP.1,3-5 The distending pressure produced by HFNC is unknown at the bedside when it is not continuously monitored. Monitoring this distending pressure is paramount, since this continuous pressure changes with changes in pulmonary mechanics, such as resistance and compliance, and it is extremely variable between patients. An inability to provide the right amount of individualized respiratory support and titrate the dis- 
tending pressures to effect will lead to failure of noninvasive ventilation. On the other hand, providing a higher than needed pressure can cause excessive end-expiratory distending pressures that can cause adverse effects, such as air leaks (pneumothorax, pneumomediastinum) and gastrointestinal perforations. ${ }^{6}$ Some of the reasons for not monitoring and titrating distending pressures tailored to individual patients might be related to the fact that measurement of intrathoracic pressures often requires special tools, it is time consuming at the bedside, and the exact optimal distending pressure for preterm infants is unknown. ${ }^{3,7}$

In this study, we sought to determine the relationship between HFNC and generated end-expiratory esophageal pressures in premature infants. We also sought to determine the feasibility of end-expiratory esophageal pressure measurement using a regular feeding tube to titrate HFNC flows and avoid both insufficient and excessive distending pressures.

\section{Methods}

The study was approved by the Institutional Review Board at Metro Health Medical Center. In this prospective observational study, the inclusion criteria consisted of premature infants $(<37$ weeks gestational age) who were felt to be stable enough to undergo the study by their treating physicians on an HFNC, who had a nasogastric or orogastric feeding tube in place, and whose parents provided written informed consent to participate in the study. The exclusion criteria consisted of infants who had facial, oral, or nasopharyngeal anomalies that precluded the placement of an orogastric or nasogastric feeding tube as well as infants who were nil per os or who did not have a feeding tube in place.

\section{Measuring Continuous Distending Pressures}

Procedures that did not require any special equipment were used in the study. End-expiratory esophageal pressure, which represents end-expiratory pressure in the esophagus, correlates with intrapleural pressure and has been used as a surrogate for measurement of continuous distending pressures applied to the airways. ${ }^{3,8,9}$ The end-expiratory esophageal pressure measurement can be achieved by using a feeding tube (nasogastric or orogastric), overlying the lower portion of the esophagus, filled with water and connected to a pressure transducer. This technique is practical and reproducible in a neonatal ICU setting, and it has been used for nearly 3 decades. ${ }^{8,9}$ The pressure detected at the tip of the water-filled feeding tube is transduced (using an arterial line setup) to the bedside monitor, and it represents the end-expiratory esophageal pressure.

\section{QUICK LOOK}

\section{Current knowledge}

Heated and humidified high-flow nasal oxygen (HFNC) is a noninvasive means of respiratory support that improves oxygenation by meeting patient demands with high inspired oxygen concentrations and low-level endexpiratory pressure. HFNC reduces the required minute ventilation by washing out the upper respiratory dead space. In neonates, the use of large bore cannula in a small naris may result in significant increases in mean and end-expiratory airway pressures.

\section{What this paper contributes to our knowledge}

In a group of premature infants receiving HFNC oxygen at 2-8 $\mathrm{L} / \mathrm{min}$, end-expiratory pressures of 2-15 $\mathrm{cm} \mathrm{H}_{2} \mathrm{O}$ were measured using esophageal manometry. There was a significant correlation between flow and end-expiratory pressures. These results suggest caution in the application of HFNC because the pressures developed can result in a risk of barotrauma and/or hemodynamic embarrassment.

The infant's existing nasogastric/orogastric tube was used for the study. Polyurethane enteral feeding tubes (Footprint Medical, San Antonio, Texas) were used to measure end-expiratory esophageal pressure. Routine nursing guidelines were used to determine the size of the feeding tube. In general, $40-\mathrm{cm}$-long enteral feeding tubes size 5 or 6 French were used for infants $<1,500 \mathrm{~g}$, and size 8 French tubes were used for infants $>1,500 \mathrm{~g}$. Before feeding and while the infant was in a quiet state, the infant was placed in a supine position. The infant's mouth was gently closed during the study procedures. ${ }^{1,10}$ The orogastric or nasogastric feeding tube was flushed initially with air and then filled with $0.2-0.5 \mathrm{~mL}$ of sterile water. Based on previous estimated measurements, the water-filled feeding tube was pulled out of the stomach by a few $\mathrm{cm}$ to reach the lower part of the esophagus. The estimated measurements of the feeding tube from the lips or nostrils to the lower esophagus were calculated based on the length of the feeding tube from the lips (for orogastric tubes) or nostrils (for nasogastric tubes) to the ear tragus to $1-2 \mathrm{~cm}$ above the xiphoid notch. The water-filled feeding tube was then connected to a transducer using a routine arterial line measurement setup, and transduction signals were verified using a BTR 24-30 pressure transducer (Biosensors International, Newport Beach, California). The pressure transducer and nasogastric tube setup were zeroed to the atmospheric pressure in the same way an arterial line is zeroed using the same routine procedure. ${ }^{11}$ The feeding 
tube was withdrawn until the typical negative tracing deflections associated with inspiration were noticed. ${ }^{9}$

After the feeding tube was transduced, the intra-esophageal pressure waves were monitored until a regular and steady breathing pattern was observed for at least 3-5 min. After reaching steady state waveforms, the displayed endexpiratory esophageal pressure values were recorded.

For each HFNC flow, the corresponding end-expiratory esophageal pressure was recorded. A stepwise, non-randomized increase or a decrease of the flow by $1 \mathrm{~L} / \mathrm{min}$ was performed every $10 \mathrm{~min}$. The HFNC flow was changed in a sequential order, rather than a random order, to reduce the chance of physiologic decompensation. To further reduce the chance of physiologic decompensation during the study, HFNC flows were altered by no more than $4 \mathrm{~L} / \mathrm{min}$ for an individual subject. For example, for an infant with initial HFNC flow of $8 \mathrm{~L} / \mathrm{min}$, the minimum flow setting used during the study was $4 \mathrm{~L} / \mathrm{min}$. The flows ranged between a minimum of $2 \mathrm{~L} / \mathrm{min}$ and a maximum of $8 \mathrm{~L} / \mathrm{min}$. Treating physicians retained the ability to limit the number of observations to $<4 /$ subject. The study procedure was stopped if the infant developed apnea, bradycardia (heart rate $<80 /$ min), oxygen desaturation (pulse oximeter $<88 \%$ ), tachypnea (breathing frequency $>60 / \mathrm{min}$ ), or significant intercostal or subcostal retractions. Subjects did not receive any sedatives during the measurement of their end-expiratory esophageal pressure.

After the measurements were done, the nasogastric or orogastric feeding tubes were pushed back into the stomach to the original site. The position of the tip of the feeding tube was verified by the nursing staff before the next feeding (by listening with a stethoscope to the area overlying the stomach while flushing the feeding tube with air).

A Vapotherm 2000i (Vapotherm, Exeter, New Hampshire) was the HFNC system used during the study. The nasal interface used during the study was the standard Vapotherm cannula, preterm/neonate size $(1.5-\mathrm{mm}$ outer diameter) that accompanies the Vapotherm 2000i. The aim of sizing the nasal cannula was to have no more than $50 \%$ occlusion at the nasal interface. Leak around the nasal interface was not specifically measured. Because HFNC was used over the recommended and approved flow range, we did not add any pressure relief devices to the HFNC system.

Respiratory distress syndrome was defined as respiratory distress with need for oxygen and classical chest radiograph findings (air bronchogram, ground glass appearance, low lung volumes) within $24 \mathrm{~h}$ after birth. ${ }^{12}$ Bronchopulmonary dysplasia was defined as a requirement for $>21 \%$ oxygen for $>28$ d. ${ }^{13}$
Table 1. Subjects' Demographics

\begin{tabular}{lc}
\hline \hline \multicolumn{1}{c}{ Parameters } & Values $(N=19)$ \\
\hline Birth weight, mean \pm SD g & $904 \pm 574$ \\
Weight at observation, mean \pm SD g & $1,458 \pm 828$ \\
Gestation at birth, mean \pm SD wks & $26.6 \pm 3.1$ \\
Gestation at observation, mean \pm SD wks & $33.3 \pm 4.9$ \\
Male sex, $n$ (\% male) & $9(47)$ \\
Ethnicity, $n(\%)$ & \\
$\quad$ Caucasian & $4(21)$ \\
African-American & $12(63)$ \\
Hispanic & $3(16)$ \\
Antenatal steroids, $n$ (\%) & $13(68)$ \\
Surfactant, $n(\%)$ & $18(95)$ \\
Apgar scores at 1 min, median (range) & $5(1-9)$ \\
Apgar scores at 5 min, median (range) & $7(1-8)$ \\
Length of invasive mechanical ventilation, & $16.8 \pm 11.2$ \\
$\quad$ mean \pm SD d & \\
HFNC setting at the time of testing, median & $5(3-8)$ \\
$\quad$ (range) & \\
$\mathrm{F}_{\mathrm{IO}}$, mean \pm SD & $0.29 \pm 0.04$ \\
\hline
\end{tabular}

\section{Statistical Analysis}

Sample size was estimated as 19 , for a correlation between HFNC setting and end-expiratory esophageal pressure of at least 0.6 , power of $80 \%$, and significance level of 0.05 . Data were expressed as mean \pm SD and as percentages. To determine the association between end-expiratory esophageal pressure and HFNC, a linear regression analysis was conducted with the end-expiratory esophageal pressure as the dependent variable and the HFNC flow as the independent variable. To determine whether there is any association between weight and end-expiratory esophageal pressure, another linear regression analysis was conducted with end-expiratory esophageal pressure as the dependent variable and with HFNC and weight as independent variables. An analysis of variance using a general linear model was conducted to determine a difference in end-expiratory esophageal pressure between subjects at different HFNC flows. To determine whether weight has an effect on end-expiratory esophageal pressure, weight was added to the general linear model analysis as a covariate. $P$ values of $<.05$ were considered statistically significant. The statistical analysis was performed using SPSS 10.0 (SPSS, Chicago, Illinois).

\section{Results}

A total of 19 premature infants were studied. Subjects' demographics are displayed in Table 1. All infants had a history of respiratory distress syndrome with evolving or established bronchopulmonary dysplasia. During the study, 93 different HFNC-generated end-expiratory esophageal 
Table 2. End-Expiratory Esophageal Pressures Generated by Different High-Flow Nasal Cannula Flows

\begin{tabular}{lcr}
\hline \hline $\mathrm{HFNC}(\mathrm{L} / \mathrm{min})$ & EEEP $\left(\mathrm{cm} \mathrm{H}_{2} \mathrm{O}\right)$ & $n$ \\
\hline 2 & $2(2-2)$ & 1 \\
3 & $4(2-5)$ & 4 \\
$4^{*}$ & $5(3-7)$ & 18 \\
$5^{*}$ & $5(5-8)$ & 18 \\
$6^{*}$ & $7(5-10)$ & 18 \\
$7^{*}$ & $8(6-13)$ & 17 \\
$8^{*}$ & $9(6-15)$ & 17
\end{tabular}

Data are expressed as median (range).

* There was a significant difference in end-expiratory esophageal pressure in infants on

4-8-L/min high-flow nasal cannula (general linear model, $P<.001$ ).

EEEP $=$ end-expiratory esophageal pressure

HFNC $=$ high-flow nasal cannula

pressure measurements were conducted. On average, each subject had a mean (range) of 5 (1-6) end-expiratory esophageal pressure measurements. Each subject had one endexpiratory esophageal pressure measurement per HFNC flow rate level documented. All subjects tolerated the procedures well.

For each level of HFNC flow, there was a range of esophageal pressures that were generated. Table 2 shows the end-expiratory esophageal pressure levels generated by different HFNC flows. An analysis of variance using a general linear model analysis was conducted to compare subjects receiving different HFNC flows ranging between 4 and $8 \mathrm{~L} / \mathrm{min}$. There was a significant difference in endexpiratory esophageal pressure in infants on $4-8-\mathrm{L} / \mathrm{min}$ HFNC (general linear model, $P<.001$ ).

To determine the association between HFNC and endexpiratory esophageal pressure, a linear regression was conducted with the end-expiratory esophageal pressure as the dependent variable and the HFNC flow as the independent variable. There was a statistically significant association between flows and esophageal pressures. Endexpiratory esophageal pressure $\left(\mathrm{cm} \mathrm{H}_{2} \mathrm{O}\right)=1.18(95 \% \mathrm{CI}$ : 1.14-1.25) $\times$ flow (L/min) with an $\mathrm{r}^{2}$ of 0.95 and $P<$ .001 (see Fig. 1). For example, for an HFNC flow of $5 \mathrm{~L} / \mathrm{min}$, the corresponding end-expiratory esophageal pressure will be $1.18 \times 5=6 \mathrm{~cm} \mathrm{H}_{2} \mathrm{O}$.

To determine whether weight has an effect on end-expiratory esophageal pressure, a linear regression analysis was conducted with end-expiratory esophageal pressure as the dependent variable and with HFNC and weight as independent variables. There was no significant correlation between weight and end-expiratory esophageal pressure. Weight had a $\beta$ coefficient of $5.8 \times 10^{-5}(P=.32)$. However, the correlation between HFNC and end-expiratory esophageal pressure persisted with a $\beta$ coefficient of $1.18(P<.001)$.

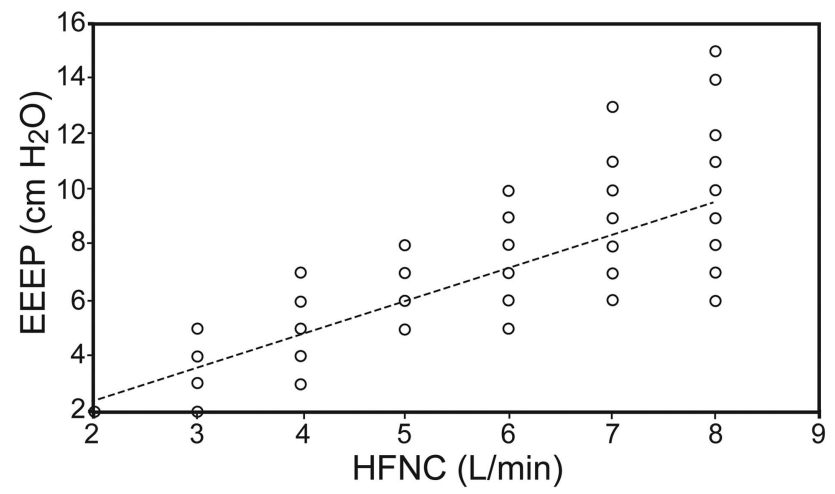

Fig. 1. Association between high-flow nasal cannula (HFNC) and generated end-expiratory esophageal pressures (EEEP) in premature infants. EEEP $=1.18 \times$ flow $\left(r^{2}=0.95 ; P<.001\right)$. Each point represents an individual EEEP measurement in a subject on a given HFNC flow.

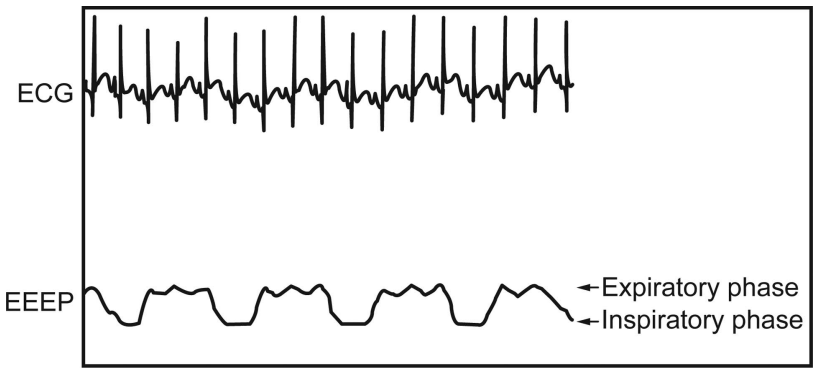

Fig. 2. A representative end-expiratory esophageal pressure waveform of $4 \mathrm{~cm} \mathrm{H}_{2} \mathrm{O}$ corresponding to a high-flow nasal cannula of $4 \mathrm{~L} / \mathrm{min}$. ECG = electrocardiogram; EEEP = end-expiratory esophageal pressure.

We also conducted a variance analysis using a general linear model to determine whether there was any difference in end-expiratory esophageal pressure at different HFNC flows ranging between 4 and $8 \mathrm{~L} / \mathrm{min}$ with weight as a covariate, and the result of our analysis showed no statistically significant effect of weight on end-expiratory esophageal pressure $(P=.72)$. A representative end-expiratory esophageal pressure measurement at $4-\mathrm{L} / \mathrm{min}$ HFNC flow is provided in Figure 2.

\section{Discussion}

We have shown in this study that there is a significant association between HFNC flow and end-expiratory esophageal pressure in premature infants. We have also shown that end-expiratory esophageal pressure measurements are feasible at the bedside by using regular nasogastric or orogastric feeding tubes. Such a technique will help practitioners to titrate HFNC flow to avoid both insufficient and excessive distending esophageal pressures.

HFNC (flows $>2 \mathrm{~L} / \mathrm{min}$ ) devices are increasingly being used with no documented benefits for clinical outcomes. 
With the use of heated and humidified air, higher flows of up to $8 \mathrm{~L} / \mathrm{min}$ have been used in newborn infants. Concern for inadvertent delivery of excessive lung distending pressure, especially in premature infants, led us to conduct this study. Our results confirm that the delivery of significant lung distending pressures (measured as end-expiratory esophageal pressure) are seen with HFNC in premature infants.

The proposed mechanism of action of HFNC is the reduction of dead space by nasopharyngeal wash out. Because of relatively larger head size, infants have larger nasopharyngeal dead space compared with older children, which reduces the efficiency of breathing and imposes additional work of breathing. ${ }^{14}$ In an experimental model, HFNC has been shown to reduce $\mathrm{CO}_{2}$ and improve oxygenation with increasing flow. In such a model, improvement of oxygenation and ventilation happened without delivery of pressure higher than nasal CPAP, thereby indicating the positive role of nasopharyngeal washout. ${ }^{2}$

Pressures generated by HFNC have been studied previously in experimental models and in infants. Studies conducted on lung models (artificial lungs and animal models) have shown that pressure delivery was directly proportional to HFNC flow and inversely affected by the amount of leak at the nasal interface. ${ }^{15,16}$ Studies in infants have shown that the amount of pressure delivered to the pharynx, and possibly to the lungs, is associated with higher flows and lower infant weight. ${ }^{1,3-5}$ These human studies have also documented significant interpatient variability in measured pressures. Lampland et $\mathrm{al}^{3}$ reported a $161-$ $1,885 \%$ interpatient coefficient of variation in their study comparing pressures generated by HFNC with nasal CPAP. Collins et al ${ }^{17}$ also showed that pressures delivered by 2 different HFNC systems were variable and unpredictable. Our study confirms the association between HFNC flow and end-expiratory esophageal pressure in premature infants. We also confirmed in our study the significant variability of high flow-generated esophageal pressures noted in previous studies. For example, in our study at $6 \mathrm{~L} / \mathrm{min}$ flow, the end-expiratory esophageal pressure ranged from 5 to $10 \mathrm{~cm} \mathrm{H}_{2} \mathrm{O}$.

Our study has limitations. We did not study the effect of open versus closed mouth on end-expiratory esophageal pressure. Similarly, we did not study the effect of leak size at the nasal interface on end-expiratory esophageal pressure. Our study included infants with wide range of size and weight at the time of the study, and it is likely that the percent occlusion at nasal interface was variable. We believe, however, that the effect of leak at the nasal interface and mouth are better standardized and studied in in vitro models. In our study, we could only standardize the leak amount by closing the mouth passively. We did not use any special equipment for the study and were therefore unable to quantify tidal volume, pressure-time product, and measures of work of breathing. We did not measure simultaneously airway or gastric pressures or thoracic volumes, and therefore positive end-expiratory pressures may not be reflective of increased end-expiratory lung volume. The end-expiratory esophageal pressure measurements were likely to have been subject to normal variations of transpulmonary pressures across the length of the esophagus. Nevertheless, the linear trend of increasing end-expiratory esophageal pressure with increasing HFNC flow was demonstrable in our observations.

\section{Conclusions}

HFNC is associated with the generation of a variable, and in some cases very high, level of lung distending pressure. This finding is especially important for very low birthweight infants who are at high risk of developing barotrauma. Therefore, caution should be used while using HFNC in preterm infants.

\section{REFERENCES}

1. Sreenan C, Lemke RP, Hudson-Mason A, Osiovich H. High-flow nasal cannulae in the management of apnea of prematurity: a comparison with conventional nasal continuous positive airway pressure. Pediatrics 2001;107(5):1081-1083.

2. Frizzola M, Miller TL, Rodriguez ME, Zhu Y, Rojas J, Hesek A, et al. High-flow nasal cannula: impact on oxygenation and ventilation in an acute lung injury model. Pediatr Pulmonol 2011;46(1):67-74.

3. Lampland AL, Plumm B, Meyers PA, Worwa CT, Mammel MC. Observational study of humidified high-flow nasal cannula compared with nasal continuous positive airway pressure. J Pediatr 2009; 154(2):177-182.

4. Wilkinson DJ, Andersen CC, Smith K, Holberton J. Pharyngeal pressure with high-flow nasal cannulae in premature infants. J Perinatol 2008;28(1):42-47.

5. Spence KL, Murphy D, Kilian C, McGonigle R, Kilani RA. Highflow nasal cannula as a device to provide continuous positive airway pressure in infants. J Perinatol 2007;27(12):772-775.

6. Jasin LR, Kern S, Thompson S, Walter C, Rone JM, Yohannan MD. Subcutaneous scalp emphysema, pneumo-orbitis and pneumocephalus in a neonate on high humidity high flow nasal cannula. J Perinatol 2008;28(11):779-781.

7. Kubicka ZJ, Limauro J, Darnall RA. Heated, humidified high-flow nasal cannula therapy: yet another way to deliver continuous positive airway pressure? Pediatrics 2008;121(1):82-88.

8. Greenough A, Morley CJ. Oesophageal pressure measurements in ventilated preterm babies. Arch Dis Child 1982;57(11):851-855.

9. Tanswell AK, Clubb RA, Smith BT, Boston RW. Individualised continuous distending pressure applied within 6 hours of delivery in infants with respiratory distress syndrome. Arch Dis Child 1980; 55(1):33-39.

10. Locke RG, Wolfson MR, Shaffer TH, Rubenstein SD, Greenspan JS. Inadvertent administration of positive end-distending pressure during nasal cannula flow. Pediatrics 1993;91(1):135-138.

11. McGhee BH, Bridges EJ. Monitoring arterial blood pressure: what you may not know. Crit Care Nurse 2002;22(2):60-79. 


\section{HFNC-Related Esophageal Pressures}

12. Sweet DG, Carnielli V, Greisen G, Hallman M, Ozek E, Plavka R, et al. European consensus guidelines on the management of neonatal respiratory distress syndrome in preterm infants: 2013 update. Neonatology 2013;103(4):353-368.

13. Jobe AH, Bancalari E. Bronchopulmonary dysplasia. Am J Respir Crit Care Med 2001;163(7):1723-1729.

14. Numa AH, Newth CJ. Anatomic dead space in infants and children. J Appl Physiol 1996;80(5):1485-1489.

15. Sivieri EM, Gerdes JS, Abbasi S. Effect of HFNC flow rate, cannula size, and nares diameter on generated airway pressures: an in vitro study. Pediatr Pulmonol 2013;48(5):506-514.

16. Chang GY, Cox CA, Shaffer TH. Nasal cannula, CPAP, and highflow nasal cannula: effect of flow on temperature, humidity, pressure, and resistance. Biomed Instrum Technol 2011;45(1):69-74.

17. Collins CL, Holberton JR, König K. Comparison of the pharyngeal pressure provided by two heated, humidified high-flow nasal cannulae devices in premature infants. J Paediatr Child Health 2013;49(7):554-556.

This article is approved for Continuing Respiratory Care Education credit. For information and to obtain your CRCE

(free to AARC members) visit

www.rcjournal.com 